${ }^{3}$ Mayday University Hospital, London, UK; ${ }^{4}$ Royal Brompton, London, UK; ${ }^{5}$ Imperial College, London, UK; ${ }^{6}$ London Chest Hospital, London, UK

Aims The CARDia trial randomised diabetic patients to coronary artery bypass grafting (CABG) or percutaneous coronary intervention (PCI) and concluded that PCI is a potentially safe and feasible alternative to CABG in selected patients with diabetes mellitus (DM) and multivessel coronary artery disease. The impact of insulin treatment on clinical outcomes after revascularisation is unclear. The present study is a sub group analysis of the CARDia trial comparing the cardiovascular outcomes at 12 months following revascularisation between the insulin treated (IT) and non-insulin treated (NIT) group. Methods 508 patients with an established diagnosis of DM and de novo coronary artery disease were identified and randomised to CABG or PCI. Of those, 316 patients were treated with oral antidiabetic medication and the rest were treated with additional subcutaneous insulin injections. Demographics, clinical presentation, history, haemodynamic parameters, anti diabetic therapy, concomitant medications, duration of DM and HBA1C were documented. Death, stroke and myocardial infarction were classified as the primary outcome events. The secondary outcome events included death, MI, Stroke, repeat revascularisation and TIMI major bleed. The clinical results of patients in the IT and NIT groups were compared.

Results There were 192 patients in the IT group (37.8\%). Asian patients constituted one fifth of the total population with a slightly higher representation $(24.5 \%$ vs $21.6 \%)$ in the NIT. The clinical severity of dyspnoea, heart rate, systolic and diastolic BP, body mass index, risk factors for coronary artery disease appeared similar in the IT and NIT groups, but more patients in the IT group had a prior MI $(30.7 \%$ vs $19.6 \%, p=0.004)$ and duration of diabetes was longer in the IT group (14 vs 6 yrs, $p<0.001$ ). For the comparison of CABG vs PCI for the primary outcome events the HR and $95 \%$ CI in the IT and NIT groups respectively were 1.66 (0.76 to 3.76 ) and 1.01 (0.51 to 2.01). For death, MI, stroke, repeat revascularisation they were 2.47 (1.18 to 5.20) in the IT and 1.41 (0.71 to 2.57) in the NIT group. The results suggest that IT patients may have a worse outcome with PCI compared to CABG, whereas no difference was found for NIT patients.

Conclusion Our data suggest that insulin treatment is a marker for higher risk for PCI when compared with CABG. Treatment with insulin rather than diabetic status alone should be considered when choosing the mode of revascularisation.

\section{SUCCESSFUL RECANALISATION OF CHRONIC TOTAL OCCLUSIONS IS ASSOCIATED WITH INCREASED LONG TERM SURVIVAL}

doi:10.1136/heartjnl-2011-300198.35

1J M Behar, ${ }^{1} \mathrm{D}$ A Jones, ${ }^{1} \mathrm{R}$ Weerackody, ${ }^{1} \mathrm{~K}$ Rathod, ${ }^{1} \mathrm{C} \mathrm{J}$ Knight, ${ }^{1} \mathrm{~A}$ K Kapur, ${ }^{1} \mathrm{~A}$ Jain, ${ }^{1} \mathrm{~A}$ Wragg, ${ }^{2} \mathrm{C}$ A Thompson, ${ }^{1} \mathrm{~A}$ Mathur, ${ }^{1} \mathrm{E}$ J Smith. ${ }^{1}$ The London Chest Hospital, Barts and the London NHS Trust, London, UK; ${ }^{2}$ Department of Cardiology, Yale University, New Haven, Connecticut, USA

Introduction Chronic total occlusion (CTO) remains a challenging lesion subset. Despite advances in equipment and expertise, many CTO patients may not be offered PCI as physicians perceive procedural success may be lower, and the anatomy is stable. The aim of this study was to investigate the impact of procedural success on mortality following CTO-PCI in a large cohort of patients in the drug eluting stent era.

Methods 6122 consecutive patients underwent elective PCI at a single centre (October 2003-May 2010), 836 (13.7\%) for CTO. Demographic and procedural data were collected at the time of intervention (Abstract 35 table 1). In-hospital MACE (myocardial infarction, urgent revascularisation, stroke or death) was documented at discharge. All cause mortality data was obtained from the Office of National Statistics via the BCIS/CCAD national audit out to 4 years (mean $2.9 \pm 1.6$ ) and stratified according to successful or unsuccessful CTO recanalisation.

Abstract 35 Table 1

\begin{tabular}{llll}
\hline & $\begin{array}{l}\text { Successful } \\
\text { (n=572) }\end{array}$ & $\begin{array}{l}\text { Unsuccessful } \\
(\mathbf{n}=\mathbf{2 6 4})\end{array}$ & p value \\
\hline Age & $62.4 \pm 0.47$ & $63.7 \pm 0.69$ & 0.1 \\
Male & $433(75.7 \%)$ & $209(79.2 \%)$ & 0.3 \\
Diabetes & $151(26.9 \%)$ & $74(28.6 \%)$ & 0.6 \\
Hypertension & $320(63.8 \%)$ & $160(66.6 \%)$ & 0.5 \\
Hypercholesterolaemia & $281(56.0 \%)$ & $147(61.2 \%)$ & 0.2 \\
Previous MI & $174(31.7 \%)$ & $94(36.4 \%)$ & 0.2 \\
Radial access & $123(21.5 \%)$ & $47(17.8 \%)$ & 0.3 \\
Femoral access & $416(72.7 \%)$ & $193(73.1 \%)$ & 0.6 \\
Dual site access (bilateral femoral & $23(4.0 \%)$ & $18(6.8 \%)$ & 0.5 \\
or radial + femoral) & & & \\
\hline
\end{tabular}

Results 572 (68.4\%) CTO procedures were successful. Coronary stents were implanted in $96.9 \%$ (mean $2.3 \pm 0.1$ stents per patient, $70 \%$ drug eluting). Prior revascularisation was more frequent among patients with unsuccessful CTO-PCI than successful; prior CABG $16.5 \%$ unsuccessful vs $7.4 \%$ successful, $(p<0.0001)$, PCI $36.0 \%$ vs $21.2 \%,(p<0.0001)$. Baseline characteristics were otherwise similar (Abstract 35 table 1). Intra-procedural complications (coronary dissection, perforation, access site (dissection, haematoma) were more frequent in unsuccessful cases $(19 \%$ (52) vs $4.1 \%$ (20) $(\mathrm{p}<0.0001)$ but did not have an impact on in-hospital MACE $(2 \%$ vs $1.8 \%, p=0.6$ ). All cause mortality was $8 \%(21)$ in the unsuccessful group and 3\% (17) in the successful group out to 4 years, (Abstract 35 figure 1). Mortality following successful CTO-PCI was similar to that of the non-CTO elective PCI group $(5.1 \%, \mathrm{p}=\mathrm{NS})$.

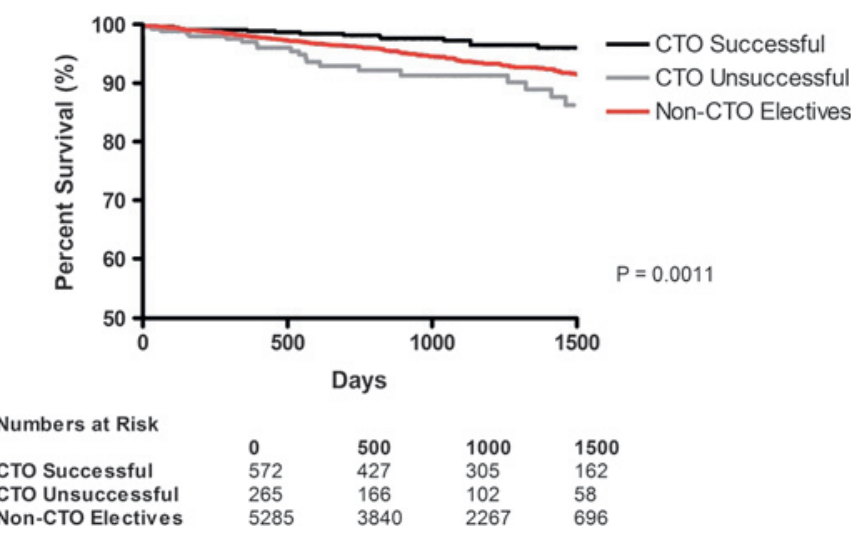

Abstract 35 Figure 1 All cause mortality after PCl for elective patients.

Conclusion A successful angiographic outcome following CTO-PCI is associated with a survival advantage out to 4 years following intervention. These data suggest that the adoption of new techniques and technologies to improve procedural success may improve prognosis.

\section{IN-STENT RESTENOSIS PRESENTS AS AN ACUTE CORONARY SYNDROME (ACS) IN 40\% OF CASES: NOT SIMPLY A BENIGN CLINICAL ENTITY}

doi:10.1136/heartjnl-2011-300198.36

H Abu-Own, E Sammut, K Rathod, L A McGill, D A Jones, A Jain, C Knight, A Mathur, A Wragg. Barts and the London NHS Trust, London, UK

Background In-stent restenosis (ISR) following stent implantation may occur in $20 \%$ to $40 \%$ of the cases according to patient and lesion complexity. Although in the past ISR used to be seen as a "benign" 
event, more recent studies suggested that a reasonable amount of patients with ISR many develop ACS as the first manifestation of this adverse event. The aim of this study was to determine the different clinical presentations of ISR in a large cohort of consecutive, nonselected patients and compare with native coronary disease.

Methods 14445 consecutive patients underwent PCI at a single centre (October 2003-May 2010), we identified 922 (6.4\%) cases presenting with restenosis after previous PCI. All patients with restenosis presented with new or recurrent symptoms. Demographic and procedural data were collected at the time of intervention (Abstract 36 table 1). In-hospital MACE (myocardial infarction, urgent revascularisation, stroke or death) was documented at discharge. All cause mortality data was obtained from the Office of National Statistics via the BCIS/CCAD national audit out to 3.2 years (mean $3.1 \pm 1.8$ years).

\section{Abstract 36 Table 1}

\begin{tabular}{llll}
\hline Total & $\begin{array}{l}\text { Restenosis } \\
\mathbf{n = 9 2 2}\end{array}$ & $\begin{array}{l}\text { Native disease } \\
\mathbf{n = 1 3 5 2 3}\end{array}$ & $\begin{array}{l}\text { Sig } \\
-\end{array}$ \\
\hline Age & 63.09 & 63.76 & 0.0868 \\
Ethnicity (cau) & $683(74.2 \%)$ & $9160(97.8 \%)$ & $\mathrm{p}<0.0001$ \\
Previous MI & $411(44.6 \%)$ & $2160(23.1 \%)$ & $\mathrm{p}<0.0001$ \\
Previous CABG & $120(13.0 \%)$ & $648(6.9 \%)$ & $\mathrm{p}<0.0001$ \\
DM & $299(32.5 \%)$ & $1986(21.2 \%)$ & $\mathrm{p}<0.0001$ \\
HTN & $545(59.1 \%)$ & $4170(44.5 \%)$ & $\mathrm{p}<0.0001$ \\
Hchol & $544(59.0 \%)$ & $3540(37.8 \%)$ & $\mathrm{p}<0.0001$ \\
Card Shock & $6(0.7 \%)$ & $100(1.1 \%)$ & 0.2339 \\
\hline
\end{tabular}

Results Restenosis presented in $60.4 \%$ as stable angina, $30.6 \%$ as unstable angina/Non-ST elevation MI and 9\% with ST-elevation Myocardial Infarction. Cardiogenic shock was reported in 6 patients $(0.65 \%)$. Women had a higher incidence of unstable angina/nonSTEMI compared with men (32.6\% vs $29.1 \%$ ) but a lower incidence of STEMI (5\% vs 10.4\%). Baseline characteristics are listed in Abstract 36 table 1 . Mortality rate was $0.98 \%$ at 30 days, $3.9 \%$ at 1 year and $8.7 \%$ at 5 years in patients with restenosis. Comparing the restenotic group with those undergoing PCI for de novo coronary artery disease, there were similar ages and incidence of cardiogenic shock but the restenotic group had higher rates of baseline risk factors (diabetes, hypertension, hyerpcholesterolaemia) and higher rates of previous CABG and MI. There was also a higher proportion of South Asians in the restenotic group. See Abstract 36 table 1. Comparing outcome measures, there were similar rates of inhospital MACE in the 2 groups and over a 5 year follow-up period, there was no difference in all cause mortality. There was no difference in outcome of patients with restenosis vs de novo coronary artery disease regardless of presentation (angina, UA/ NSTEMI/STEMI). See Abstract 36 figures 1 and 2.

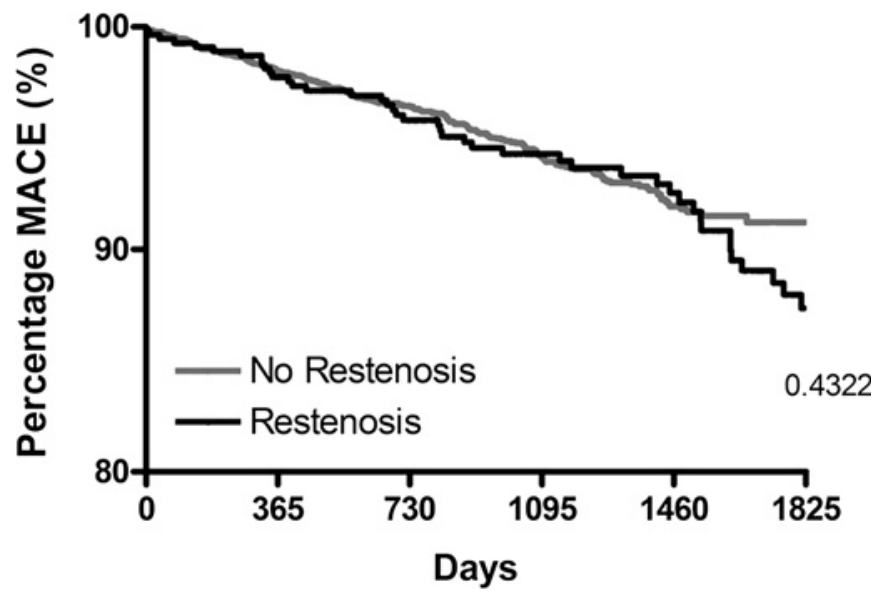

Abstract 36 Figure 1 Comparison of mortality between restenosis and no restenosis in STABLE.

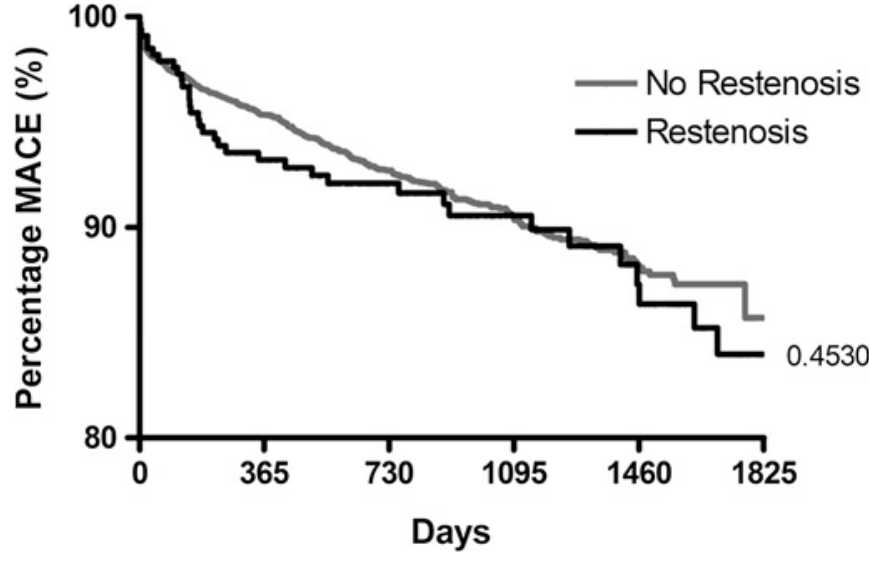

Abstract 36 Figure 2 Comparison of mortality between restenosis and no restenosis in ACS.

Conclusions Clinical in-stent restenosis can frequently present as MI and such patients are more likely to have an aggressive angiographic pattern of restenosis. Drug-eluting stents with improved designs or drug elution systems that further decrease the incidence of ISR are needed.

\section{DECREASE IN MACE RATES ASSOCIATED WITH DRUG ELUTING STENT USE IN PATIENTS WITH DIABETES UNDERGOING PCI IN LARGE DIAMETER CORONARY ARTERIES}

doi:10.1136/heartjnl-2011-300198.37

${ }^{1} \mathrm{~A}$ Dixit, ${ }^{2} \mathrm{~S}$ Nair, ${ }^{2} \mathrm{P}$ Williams, ${ }^{2} \mathrm{~A}$ Wiper, ${ }^{2} \mathrm{~B}$ Clarke, ${ }^{2} \mathrm{C}$ Deaton, ${ }^{2} \mathrm{M}$ El-Omar, ${ }^{2} \mathrm{D}$ Fraser, ${ }^{2} \mathrm{R}$ Khattar, ${ }^{2} \mathrm{~V}$ Mahadevan, ${ }^{2} \mathrm{~L}$ Neyses, ${ }^{2} \mathrm{~F}$ Ordoubadi, ${ }^{2} \mathrm{M}$ Mamas. ${ }^{1}$ University Hospital Manchester MHC, Manchester, UK; ${ }^{2}$ Manchester Royal Infirmary, Manchester, UK

Introduction Both large multi centre trials and registry studies have demonstrated that PCI with drug eluting stents (DES) is associated with reduced MACE and restenosis rates compared to bare metal stents (BMS) in native coronary vessels, although this benefit is less evident in those patients with a larger coronary vessel diameter and MACE rates may actually paradoxically increase in this cohort as observed in the BASKET trial. In diabetic patients, a similar or even greater absolute reduction in MACE rates / restenosis risk is seen associated with DES use, although it is unclear as to whether any benefit persists in those with larger diameter native coronary vessels. Previous data derived from diabetic patients in large diameter native coronary vessels has come from registry studies in which numbers were either small ( $<200$ patients) or were from highly selected patient sub groups excluding high-risk individuals (SCAAR registry). Methods We therefore retrospectively studied 1165 consecutive diabetic patients with target vessel diameter $\geq 3 \mathrm{~mm}$ admitted to our centre for PCI from 2003 to 2009, the largest series of its kind to date. Primary endpoint was defined as total mortality and secondary endpoint was major adverse cardiac event (MACE) defined as composite endpoint of Death, Stroke, MI, Stent Thrombosis and Target Lesion / Vessel Re-Vascularisation.

Results Of the 1165 patients studied, 170 had BMS and 995 had DES. Mean follow-up period was $43.3 \pm 21.8$ months (median 41.8 months). $73.5 \%$ were male in the BMS cohort vs $73.1 \%$ in the DES cohort $(p>0.05)$. Mean age was $62.8 \pm 11.2$ in BMS and $62.3 \pm$ 10.4 years old in DES ( $p=0.55)$. Other demographic parameters were similar in both groups. There were a total of $23 / 170$ deaths in BMS cohort $(13.5 \%)$ and $91 / 995$ in DES cohort (9.1\%), (HR 1.38; $95 \% \mathrm{CI}$ 0.83 to $2.27, \mathrm{p}=0.21)$. A total of $42 / 170(24.7 \%)$ and $163 / 995(16.3 \%)$ MACE events were observed in the BMS and DES cohort respectively (HR 1.49; 1.02 to $2.19, \mathrm{p}=0.04$ ). Multivariate analysis 